\title{
Facial morphological characteristics of mouth breathers vs. nasal breathers: A systematic review and meta-analysis of lateral cephalometric data
}

\author{
WEIYING ZHENG，XI ZHANG，JIAZENG DONG and JIANMING HE \\ Department of Stomatology, The Second Affiliated Hospital of Jiaxing University, Jiaxing, Zhejiang 314000, P.R. China
}

Received September 23, 2019; Accepted January 30, 2020

DOI: $10.3892 / \mathrm{etm} .2020 .8611$

\begin{abstract}
The present systematic review and meta-analysis was performed to assess the association between mouth breathing and facial morphological characteristics in children and adolescents. PubMed, Medline, Scopus and Google Scholar databases were searched for cross-sectional case-control studies published between 1st January 1980 and 1st April 2019. Studies comparing cephalometric data of mouth breathers and nasal breathers were included, while studies on adults were excluded. Meta-analysis was performed regarding 11 angular and 4 linear measurements. A total of 19 studies were included in the systematic review and data from 18 studies were extracted for the meta-analysis. The results indicated statistically significant decreases in Sella-Nasion-Point A (SNA) angle [mean difference (MD) $=-1.33$; 95\% CI -2.03 to $-0.63 ; \mathrm{P}=0.0002$ ] and Sella-Nasion-Point $\mathrm{B}$ (SNB) angle of mouth breathers as compared to nasal breathers $(\mathrm{MD}=-1.33$; 95\% CI -2.18 to $-0.49 ; \mathrm{P}=0.002)$. There was no difference in Point A-Nasion-Point B (ANB) angle between the two groups $(\mathrm{MD}=0.25 ; 95 \% \mathrm{CI}-0.26$ to $0.75 ; \mathrm{P}=0.34)$. Mouth breathers demonstrated an increased mandibular plane angle, total and lower anterior facial height and decreased posterior facial height. Within the limitations of the study, the results indicated that mouth breathers tended to have a retrognathic maxilla and mandible, vertical growth pattern with high mandibular plane angle, downward and backward rotation of the mandible and an increase in total and lower anterior facial height and decrease in posterior facial height. Further high-quality studies are required to strengthen the evidence on this subject.
\end{abstract}

Correspondence to: Dr Weiying Zheng, Department of Stomatology, The Second Affiliated Hospital of Jiaxing University, 1518 Huancheng North Road, Jiaxing, Zhejiang 314000, P.R. China E-mail: zhengwy8986@163.com

Key words: mouth breathing, nasal obstruction, growth and development, cephalometry

\section{Introduction}

The influence of nasal or oral breathing patterns on craniofacial growth and development has been widely debated in the orthodontic literature (1). According to Moss's functional matrix theory, normal nasal respiratory function is necessary for the balanced growth of craniofacial structures. Nasal respiration along with other functions of the craniofacial complex, e.g., mastication and swallowing, influence the amount and direction of craniofacial growth (2). Mouth breathing (MB) may result in several functional transformations, including changes in tongue position, as well as the oral and peri-oral muscular balance. Studies on MB have also reported changes in the posture of head and neck, which appears to facilitate oral breathing by increasing airflow through the upper airway (3).

The etiology of MB may be multifactorial and attributable to anatomic factors, including narrow airways, adenotonsillar hypertrophy, nasal septal deviation, nasal polyps, respiratory allergies, nasal turbinate hypertrophy and sleep position (4). Irrespective of the cause, chronic MB results in several morphological changes and is known to cause 'adenoid facies' (5). It is characterized by a narrow upper dental arch, retroclined mandibular incisors, an incompetent lip seal, a steep mandibular plane angle and an increased anterior facial height (6). Several studies have analyzed the facial morphological characteristics of mouth breathers and compared them with a cohort of nasal breathers (4,7-10). While the majority of studies indicated the presence of significant facial morphological changes in mouth breathers, others have failed to elicit direct evidence of an association between respiratory patterns and specific facial skeletal changes or malocclusions (10-12). Literature is also devoid of comprehensive level-1 evidence in the form of a systematic review and meta-analysis to firmly establish any cause-effect association. The aim of the present study was, therefore, to perform a systematic literature search and analyze available evidence to assess the difference in facial characteristics of children and adolescents who are mouth breathers or nasal breathers.

\section{Materials and methods}

Inclusion and exclusion criteria. The present systematic review was performed following the Preferred Reporting of Items 
for Systematic reviews and Meta-Analyses guidelines (13). A search was performed for cross-sectional, case-control studies reporting cephalometric measures of mouth-breathing individuals. The inclusion criteria were as follows: i) Studies performed on children and adolescents. ii) Studies comparing cephalometric data with a control group of nasal breathers. ii) Studies not including participants who had undergone surgery for airway obstruction or had undergone orthodontic treatment.

Studies that were performed on syndromic individuals or a cohort of specific malocclusion (e.g., studies performed on class 2 division 1 malocclusion) were excluded. Studies reporting incomplete data, uncontrolled studies, those published in a language other than English, case reports, abstracts and review papers were also excluded.

Search strategy and data extraction. Two reviewers independently searched the PubMed, Medline via OVID, Scopus, Web of Science and Google Scholar databases for studies published from 1st January 1980 to 1st April 2019. The key-words used in various combinations were as follows: 'Mouth breathers'; 'nasal breathers'; 'facial morphology'; 'facial characteristics'; 'growth pattern'; 'malocclusion'; 'dentofacial'; 'skeletal' and 'cephalometric'. The search strategy and results are provided in Table SI. Studies were initially evaluated at the title and abstract level. Full-text manuscripts of relevant entries were analyzed further based on the inclusion/exclusion criteria. Any disagreement was resolved by discussion. References of included studies and review articles were manually searched to identify additional articles.

Data were extracted by two authors independently and included the following: Authors' names, country of origin, year of publication, study design, etiology and diagnosis of $\mathrm{MB}$, number of participants, and demographic and cephalometric data. Corresponding authors were contacted via email for missing data.

Risk of bias assessment. The assessment criteria from a previous meta-analysis from 2013 (14) on patients with obstructive sleep apnea were modified and used for evaluation of the quality of the studies included. Articles were analyzed independently based on 5 criteria relevant to clinical research in his area. Studies were rated as 'Yes', 'No' or 'Unclear' on the following questions: i) Was the control group appropriately matched? ii) Were diagnostic criteria for MB adequately defined? iii) Was the reliability of cephalometric tracing measured? iv) Was the cephalometric evaluator masked? v) Were the cephalometric landmarks used in the study pre-defined?

Studies were initially scored by two reviewers independently. The scores were later matched and any disagreement resolved by discussion.

Statistical analysis. Meta-analysis was performed only if at least 3 studies reported similar cephalometric data. Mean and standard deviation of the scores of cephalometric values were used for estimating the pooled effect size. Review Manager [RevMan, version 5.3; 2014; Nordic Cochrane Centre (Cochrane Collaboration)] was used for the analysis. Heterogeneity was calculated using the $\mathrm{I}^{2}$ statistic. $\mathrm{I}^{2}$ values
$<25 \%$ indicated no heterogeneity, $25-50 \%$ represented low heterogeneity, values of $50-75 \%$ medium and $>75 \%$ represented substantial heterogeneity. However, even in cases of low or no heterogeneity, a random-effects model was used for the meta-analysis due to heterogeneities that exist in the samples and other geographical and methodological variations amongst the included studies. The influence of each study on the pooled effect size was analyzed using a sensitivity analysis. By using the one-study-out method, it was assessed whether deleting each individual study significantly changed the pooled results of the meta-analysis.

\section{Results}

Search outcome. A total of 2,510 relevant entries were initially identified using the search strategy (Fig. 1). Of these, 28 articles were eligible for full-text review. Subsequently, 9 studies were excluded as 2 were duplicate publications $(15,16), 2$ studies did not report cephalometric data $(17,18)$, mean and SD scores of cephalometric variables were not available in 1 study (19), orthodontic treatment was ongoing in 1 study (20), only soft tissue cephalometric variables were studied in 1 article (21), 1 study did not have a control group (5) and 1 study included adult participants as well (22). A total of 19 articles were finally included (3,4,6-10,23-34).

Characteristics of included studies. Details of the studies included are presented in Table I. A total of 7 studies were performed in Brazil (3,10,24-26,32,34), 3 in Spain $(7,28,30)$, 2 in the US (23,31), 2 in Italy (9,33), and 1 each in India (27), Saudi Arabia (8), Morocco (29), Israel (4) and Turkey (6). All were cross-sectional studies comparing cephalometric data of mouth breathers with age and a gender-matched control group of nasal breathers. MB was diagnosed using a questionnaire filled by parents of participants, using clinical history and examination and/or nasopharyngoscopy. The majority of the included studies did not specify the cause of MB. The etiology of MB was exclusively allergic rhinitis in 3 studies $(7,23,31)$ and nasal septum deviation in one study (33). The age group of the included sample varied across studies. A total of 2 studies reported separate data sets for two age-groups $(29,30)$. These sub-groups were pooled separately for quantitative analysis. Another study used two control groups, one of the non-allergic siblings and one historic control group (23). For the meta-analysis, data of the non-allergic sibling group was included.

Risk of bias assessment. A detailed risk of bias assessment of the studies included is presented in Table II. Only 1 study provided a statistical analysis of baseline similarity amongst the study and control groups (26). The skeletal maturation status of nose and mouth breathers was reported by 1 study (34). Furthermore, 2 studies diagnosed MB based on history and parental reporting only $(9,26)$, while one did not specify the method of diagnosis (29). A total of 8 studies reported on the reliability of cephalometric tracing by error analysis $(6,9,10,23,30,31,33,34)$. Only 2 studies performed a blinded examination of the radiograph by the cephalometric evaluator $(26,28)$. Cephalometric landmarks were pre-defined in all except 1 study (27). 


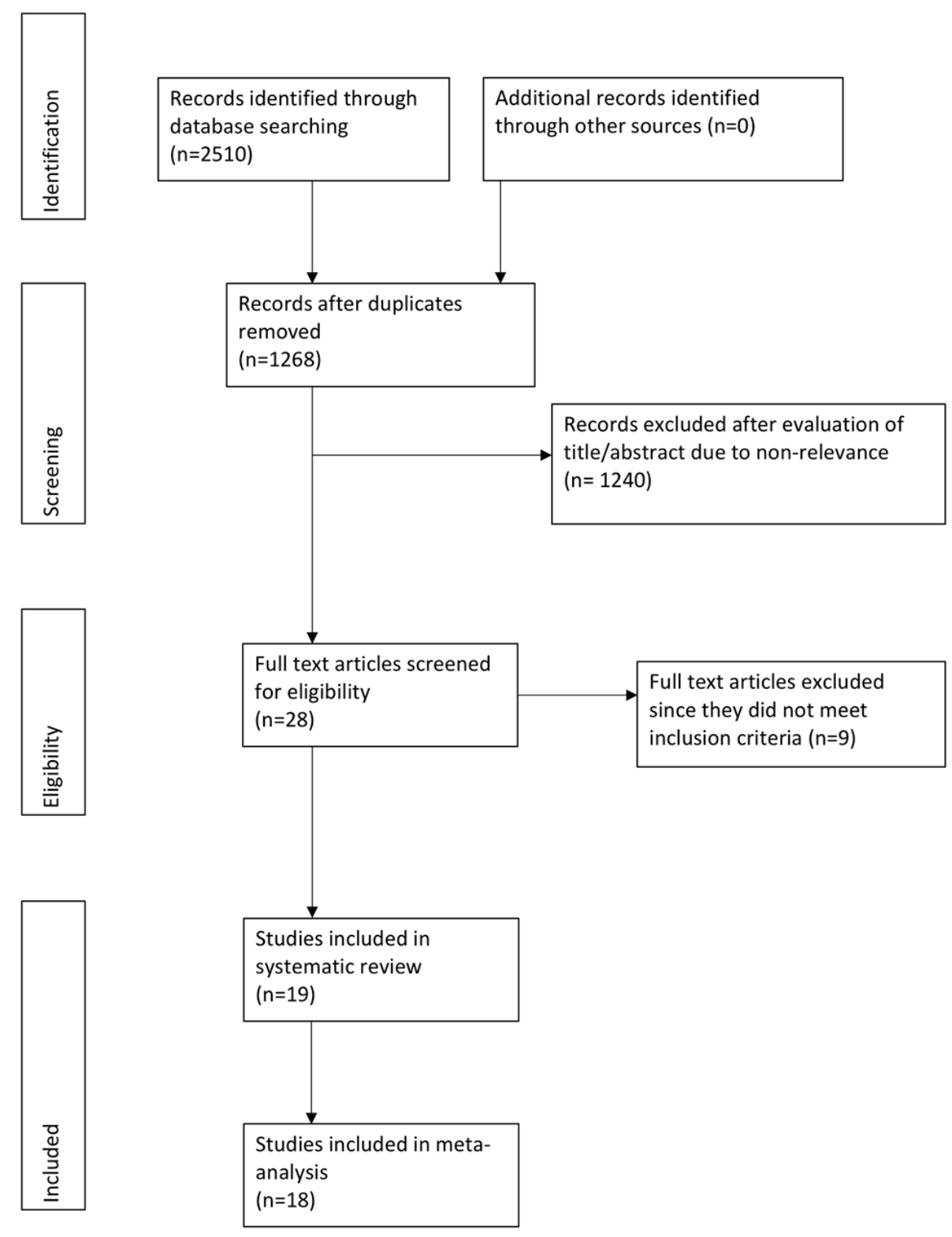

Figure 1. Study flow chart.

Meta-analysis. Cephalometric variables reported by individual studies were reviewed and, based on the frequency of reporting, a total of 11 angular and 4 linear measurements were selected for meta-analysis. The different measurements are defined in Table III. Since the majority of studies assessed facial characteristics on lateral cephalograms, data from the postero-anterior cephalogram were not included in the meta-analysis. Furthermore, 1 study did not analyze any of the 16 variables and was therefore not included in the meta-analysis (8). Missing data of 1 study were provided by the corresponding author via email (30).

Data from 13 studies, comprising 606 mouth breathers and 626 nasal breathers, were pooled for the angular measurement SNA (Table III). Meta-analysis indicated a statistically significant decrease in the SNA angle of mouth breathers as compared with that of nasal breathers [random-effects model: Mean difference $(\mathrm{MD})=-1.33 ; 95 \% \mathrm{CI},-2.03$ to $-0.63 ; \mathrm{P}=0.0002 ; \mathrm{I}^{2}=69 \%$; Fig. 2]. Similarly, pooled data of 740 mouth breathers and 744 nasal breathers indicated a reduced SNB angle (Table III) in mouth breathers (random-effects model: $\mathrm{MD}=-1.40 ; 95 \% \mathrm{CI}$, -2.20 to $-0.60 ; \mathrm{P}=0.0006 ; \mathrm{I}^{2}=81 \%$; Fig. 3). A meta-analysis of data from 503 mouth breathers and 524 nasal breathers did not indicate any significant difference in the ANB angle (Table III) between the 2 groups (random-effects model: $\mathrm{MD}=0.36$; $95 \%$ CI, -0.13 to $0.85 ; \mathrm{P}=0.15 ; \mathrm{I}^{2}=74 \%$; Fig. 4 ).

Different mandibular plane angles were pooled separately. Mandibular plane angles of SN.GoGn (random-effects model: $\mathrm{MD}=3.38$; $95 \% \mathrm{CI}, 2.77$ to $3.98 ; \mathrm{P}<0.00001 ; \mathrm{I}^{2}=2 \%$; Fig. $5 \mathrm{~A}$ ) and SN.MP (random-effects model: $\mathrm{MD}=4.02 ; 95 \% \mathrm{CI}, 2.60$ to 5.44 ; $\mathrm{P}<0.00001 ; \mathrm{I}^{2}=62 \%$; Fig. 5B; Table III) were significantly increased in mouth breathers. However, meta-analysis of data from 4 studies did not reveal any significant difference in FMA (random-effects model: $\mathrm{MD}=0.93 ; 95 \% \mathrm{CI},-0.59$ to 2.45; $\mathrm{P}=0.23 ; \mathrm{I}^{2}=51 \%$; Fig. 5C; Table III).

As indicated in Fig. 6, there was a significant increase in the angles NSGn (random-effects model: $M D=2.49$; 


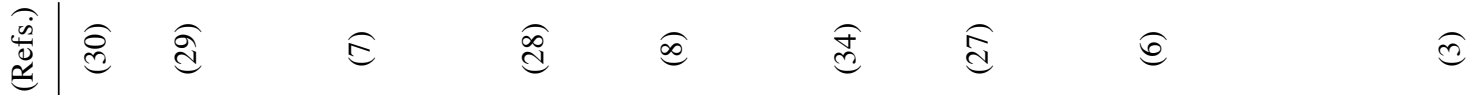

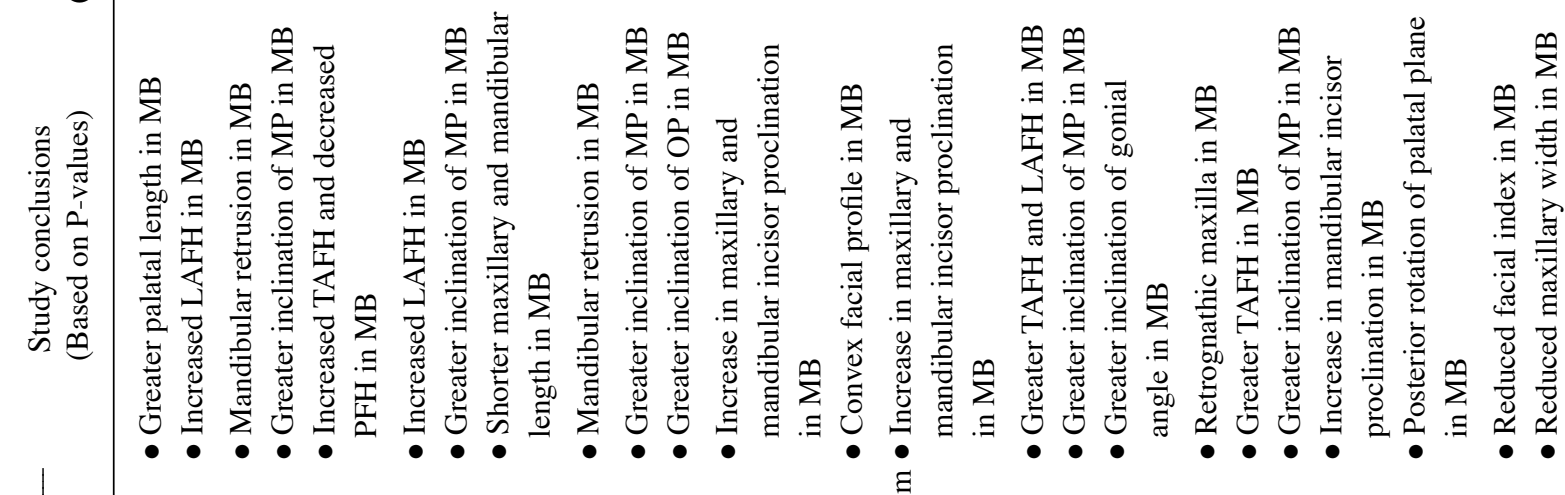

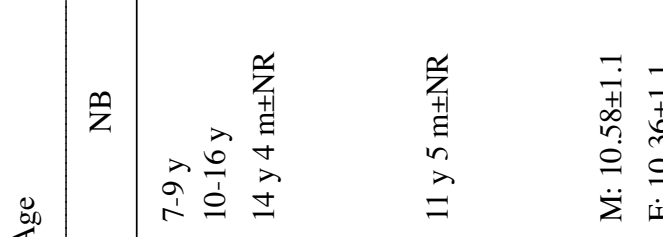

$\varangle$

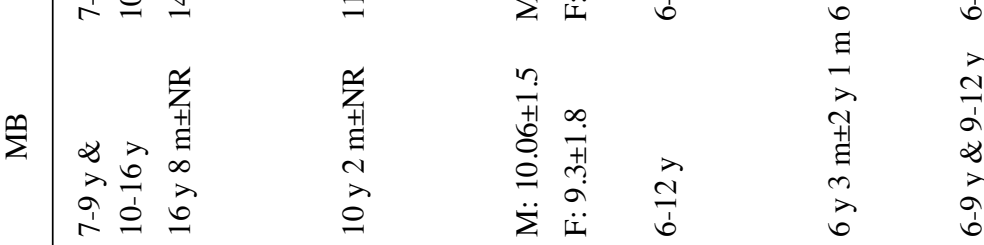

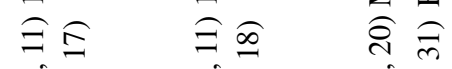

ถิธ

$\overline{\mathrm{g}}$

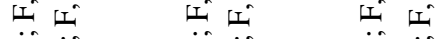

庄

$\rightarrow$ त

$\ddot{\sim} \ddot{b}$

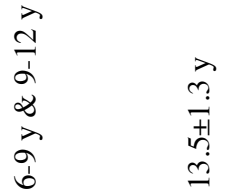

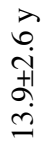

$\sum$

$\ddot{\dot{n}} \ddot{\mathrm{n}}$

$\sum$

$\ddot{\sim} \ddot{z} \tilde{z}$

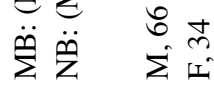

유규요

$m$

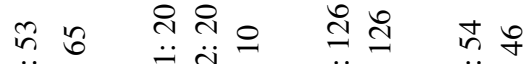

$\stackrel{\substack{n \\+1 \\+\infty}}{\infty}$

$\stackrel{\substack{i \\ \stackrel{i+1}{i}}}{i}$

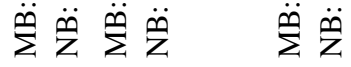

芫

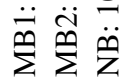

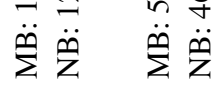

$\stackrel{2}{\underline{\infty}}$

$\underset{0}{\stackrel{1}{7}}$

₹ิ

$\ddot{\circ} \dot{\infty}$

$\sum \dot{\varepsilon}$

$\ddot{\dot{v}} \ddot{\mathrm{Z}}$

$\ddot{m}$

$\sum \sum$

$\ddot{m} \ddot{z}$

$\because ?$

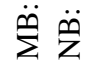

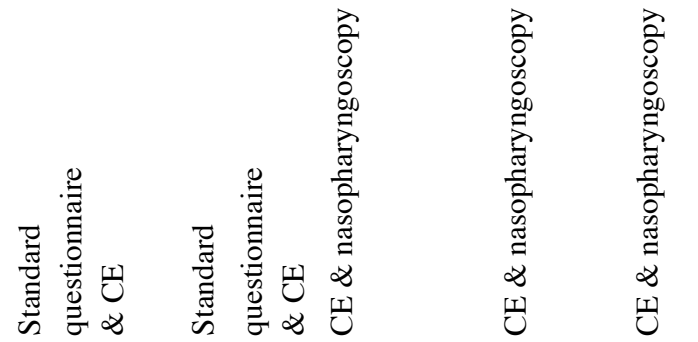

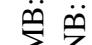
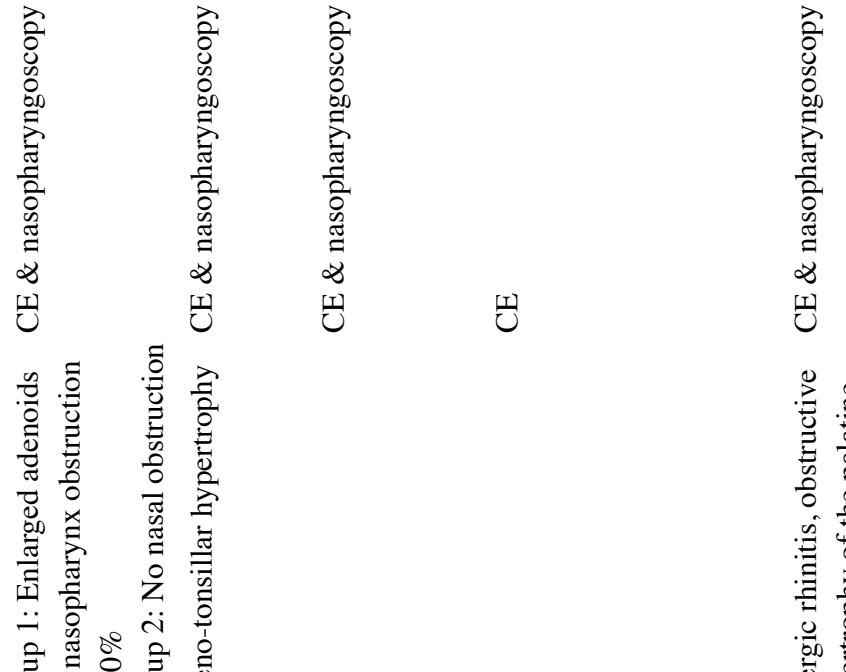

떵

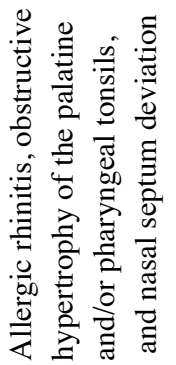

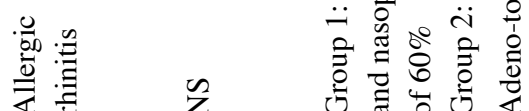

$\tilde{z}$

$\bar{z}$

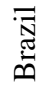

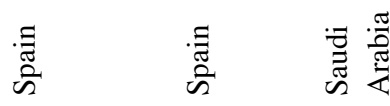

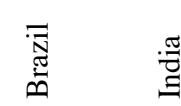

害

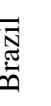

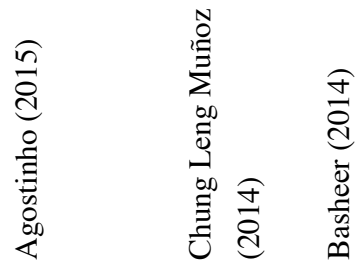

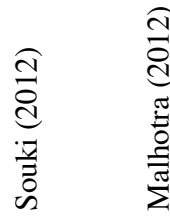

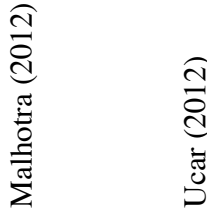

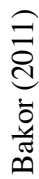




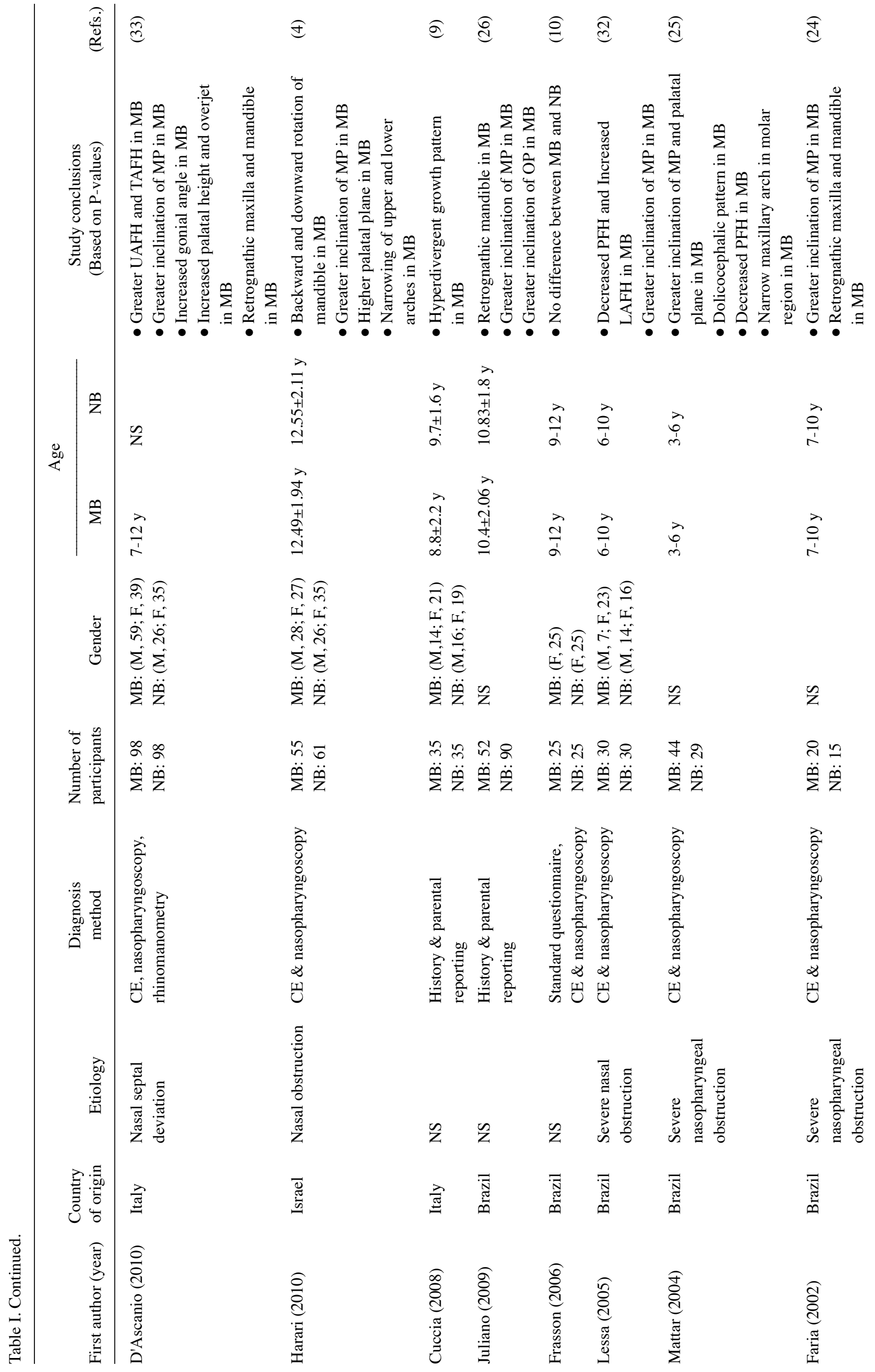


95\% CI, 1.09 to 3.89; $\mathrm{P}=0.0005 ; \mathrm{I}^{2}=77 \%$; Fig. 6A; Table III), SN.OP (random-effects model: $\mathrm{MD}=2.78 ; 95 \% \mathrm{CI}, 1.80$ to 3.75 ; $\mathrm{P}<0.00001 ; \mathrm{I}^{2}=0 \%$; Fig. $6 \mathrm{C}$; Table III) and MP.PP (random-effects model: $\mathrm{MD}=4.19 ; 95 \% \mathrm{CI}, 3.10$ to 5.29; $\mathrm{P}<0.00001 ; \mathrm{I}^{2}=67 \%$; Fig. 6D; Table III) in the mouth-breathing group. No significant difference was identified between the two groups for the angular variable SN.PP (random-effects model: $\mathrm{MD}=-0.45 ; 95 \% \mathrm{CI},-1.51$ to $0.62 ; \mathrm{P}=0.41 ; \mathrm{I}^{2}=20 \%$; Fig. 6B; Table III). The gonial angle (ArGoMe; Table III) was significantly increased in mouth breathers as compared to nasal breathers (random-effects model: $\mathrm{MD}=2.11 ; 95 \% \mathrm{CI}$, 0.41 to $3.81 ; \mathrm{P}=0.02 ; \mathrm{I}^{2}=88 \%$; Fig. $6 \mathrm{E}$ ).

Total anterior facial height $(\mathrm{N}-\mathrm{Me}$; random-effects model: $\mathrm{MD}=4.16 ; 95 \% \mathrm{CI}, 2.23$ to $6.08 ; \mathrm{P}<0.0001 ; \mathrm{I}^{2}=80 \%$; Fig. 7A; Table III) and lower anterior facial height (ANS-Me; random-effects model: $\mathrm{MD}=3.70 ; 95 \% \mathrm{CI}, 1.44$ to 5.96; $\mathrm{P}=0.001 ; \mathrm{I}^{2}=84 \%$; Fig. 7B; Table III) were significantly greater in mouth breathers. Linear measurements of Ar-Go (random-effects model: $\mathrm{MD}=-1.39 ; 95 \% \mathrm{CI},-1.93$ to -0.85 ; $\mathrm{P}<0.00001 ; \mathrm{I}^{2}=0 \%$; Fig. 7C) and S-Go (random-effects model: $\mathrm{MD}=-1.50 ; 95 \%$ CI, -2.35 to $-0.65 ; \mathrm{P}=0.0005 ; \mathrm{I}^{2}=0 \%$; Fig. 7D; Table III), denoting posterior facial height, were reduced in mouth breathers as compared to nasal breathers.

Sensitivity analysis. Sensitivity analysis was performed by removing each study sequentially from the meta-analysis to assess any changes in results. No change in the significance of any variable was identified in the sensitivity analysis.

\section{Discussion}

The primary objective of the present review was to evaluate facial morphological differences in mouth-breathing children and adolescents as compared to age and gender-matched control groups of nasal breathers. Considering the multiple different angulars and linear measurements evaluated by the studies included, it was not possible to pool all variables for a meta-analysis. The variables used in the present meta-analysis may be broadly divided as follows: Those measuring the association of maxilla and mandible to the cranial base (SNA and SNB) and each other (ANB), mandibular plane angles (SN.GoGn, FMA and SN.MP), Y-axis angle (NSGn), association of occlusal and palatal planes to the cranial base and mandible (SN.OP, SN.MP and MP.PP), the gonial angle (ArGoMe) and anterior and posterior facial height measurements (N-Me, ANS-Me, Ar-Go and S-Go).

The association of the maxilla with the cranial base was measured in 14 studies. Of these studies, 8 individually did not report any significant retrusion of the maxilla in their study population $(4,7,9,10,25,28-30)$. Similarly, data on the association of the mandible to the cranial base were reported by 15 studies. Individually, the results of these studies were divided, with 7 reporting a significant difference in SNB angles as compared to controls $(4,6,24,27,28,31,34)$ and 8 studies indicating no such difference $(3,7,9,23,25,26,29)$. Concerning etiology, while Bresolin et al (31), in a cohort of patients with allergic rhinitis, revealed retrusion of maxilla and mandible, Agostinho et al (7) did not identify any significant change in anteroposterior maxilla-mandibular position in mouth breathers with the same etiology. There were also no apparent 
Table II. Quality assessment of included studies.

\begin{tabular}{|c|c|c|c|c|c|c|}
\hline First author (year) & $\begin{array}{l}\text { Was the } \\
\text { control group } \\
\text { appropriately } \\
\text { matched? }\end{array}$ & $\begin{array}{l}\text { Were diagnostic } \\
\text { criteria for } \\
\text { mouth breathing } \\
\text { adequately defined? }\end{array}$ & $\begin{array}{c}\text { Was } \\
\text { reliability of } \\
\text { cephalometric } \\
\text { tracing measured? }\end{array}$ & $\begin{array}{c}\text { Was the } \\
\text { cephalometric } \\
\text { evaluator masked? }\end{array}$ & $\begin{array}{l}\text { Were the } \\
\text { cephalometric } \\
\text { landmarks } \\
\text { predefined? }\end{array}$ & (Refs.) \\
\hline Chambi-Rocha (2018) & Unclear & Yes & Yes & No & Yes & $(30)$ \\
\hline El Aouame (2016) & Unclear & Unclear & No & No & Yes & (29) \\
\hline Agostinho (2015) & Unclear & Yes & Unclear & No & Yes & (7) \\
\hline $\begin{array}{l}\text { Chung Leng Muñoz } \\
\text { (2014) }\end{array}$ & Unclear & Yes & No & Yes & Yes & (25) \\
\hline Basheer (2014) & Unclear & Yes & No & No & Yes & $(8)$ \\
\hline Souki (2012) & Yes & Yes & Yes & No & Yes & (34) \\
\hline Malhotra (2012) & Unclear & Yes & No & No & No & (27) \\
\hline Ucar (2012) & Unclear & Yes & Yes & No & Yes & (6) \\
\hline Bakor (2011) & Unclear & Yes & No & No & Yes & (3) \\
\hline D'Ascanio (2010) & Unclear & Yes & Yes & No & Yes & (33) \\
\hline Harari (2010) & Unclear & Yes & No & No & Yes & (4) \\
\hline Cuccia (2008) & Unclear & No & Yes & No & Yes & (9) \\
\hline Juliano (2009) & Yes & No & No & Yes & Yes & (26) \\
\hline Frasson (2006) & Unclear & Yes & Yes & No & Yes & (10) \\
\hline Lessa (2005) & Unclear & Yes & No & No & Yes & (32) \\
\hline Mattar (2004) & Unclear & Yes & No & No & Yes & (25) \\
\hline Faria (2002) & Unclear & Yes & No & No & Yes & (24) \\
\hline Trask (1987) & Unclear & Yes & Yes & No & Yes & (23) \\
\hline Bresolin (1983) & Unclear & Yes & Yes & No & Yes & (31) \\
\hline
\end{tabular}

Table III. Abbreviations of cephalometric variables used for meta-analysis.

A, Angular measurements

\begin{tabular}{ll}
\hline Variable & \\
\hline SNA & Angle between lines S-N and N-A \\
SNB & Angle between lines S-N and N-B \\
ANB & Angle between lines NA and NB \\
SN.GoGn & Angle between lines SN and Steiner's mandibular plane (line from Go to Gn) \\
FMA & Frankfort's mandibular plane angle. Angle between Frankfort's horizontal plane and Downs mandibular plane \\
& (line from Me to Go) \\
SN.MP & Angle between SN and Downs MP \\
NSGn & Angle between SN and Y axis (line from S to Gn) \\
SN.PP & Angle between SN and palatal plane (line from ANS to posterior nasal spine) \\
SN.OP & Angle between SN and occlusal plane \\
MP.PP & Angle between MP and palatal plane \\
ArGoMe & Gonial angle of mandible (angle from the Ar to Go to Me)
\end{tabular}

B, Linear measurements

\begin{tabular}{ll}
\hline Variable & \multicolumn{1}{c}{ Description } \\
\hline N-Me & Total anterior facial height. Linear distance between N and Me \\
ANS-Me & Lower anterior facial height. Linear distance between ANS and Me \\
Ar-Go & Linear distance between Ar and Go \\
S-Go & Linear distance between S and Go \\
\hline S, sella; N, nasion, A, N-point A; B, N-point B; Go, gonion; Gn, gnathion; Me, menton; ANS, anterior nasal spine; Ar, articulare; MP, mandibular \\
plane.
\end{tabular}




\begin{tabular}{|c|c|c|c|c|c|c|c|c|c|c|c|c|}
\hline \multirow[b]{2}{*}{ Study or Subgroup } & \multicolumn{3}{|c|}{ Mouth breathers } & \multicolumn{3}{|c|}{ Nasal breathers } & \multirow[b]{2}{*}{ Weight } & \multicolumn{2}{|l|}{ Mean Difference } & \multirow{2}{*}{\multicolumn{3}{|c|}{$\begin{array}{c}\text { Mean Difference } \\
\text { IV, Random, } 95 \% \mathrm{Cl}\end{array}$}} \\
\hline & Mean & SD & Total & Mean & SD & Total & & IV, Random, 95\% CI & Year & & & \\
\hline Bresolin et al & 79.4 & 3.5 & 30 & 81.7 & 3.2 & 15 & $5.4 \%$ & $-2.30[-4.35,-0.25]$ & 1983 & & & \\
\hline Faria et al & 80.6 & 3.53 & 20 & 83.13 & 2.26 & 15 & $5.7 \%$ & $-2.53[-4.45,-0.61]$ & 2002 & & & \\
\hline Mattar et al & 82.4 & 3.84 & 44 & 82.7 & 4.48 & 29 & $5.5 \%$ & $-0.30[-2.29,1.69]$ & 2004 & & & \\
\hline Frasson et al & 82.84 & 3.46 & 25 & 83.54 & 2.91 & 25 & $6.1 \%$ & $-0.70[-2.47,1.07]$ & 2006 & & & \\
\hline Cuccia et al & 81.74 & 4.45 & 35 & 80.92 & 4.29 & 35 & $5.4 \%$ & $0.82[-1.23,2.87]$ & 2008 & & & \\
\hline Juliano et al & 82.6 & 3.8 & 52 & 84.1 & 4.1 & 90 & $7.3 \%$ & $-1.50[-2.84,-0.16]$ & 2009 & & & \\
\hline D'Ascanio et al & 79.01 & 3.69 & 98 & 81.78 & 1.67 & 98 & $8.8 \%$ & $-2.77[-3.57,-1.97]$ & 2010 & & & \\
\hline Harari et al & 78.61 & 6.61 & 55 & 80.35 & 7.88 & 61 & $4.1 \%$ & $-1.74[-4.38,0.90]$ & 2010 & & & \\
\hline Malhotral et al & 78.91 & 1.99 & 28 & 82.29 & 2.05 & 34 & $8.3 \%$ & $-3.38[-4.39,-2.37]$ & 2012 & & & \\
\hline Ucar et al & 78.5 & 3.29 & 34 & 80.42 & 2.71 & 32 & $7.0 \%$ & $-1.92[-3.37,-0.47]$ & 2012 & & & \\
\hline Malhotra et al & 77.15 & 1.68 & 18 & 78.17 & 1.4 & 20 & $8.3 \%$ & $-1.02[-2.01,-0.03]$ & 2012 & & & \\
\hline Munoz et al & 82.3 & 3.7 & 53 & 83.4 & 3.8 & 65 & $7.3 \%$ & $-1.10[-2.46,0.26]$ & 2014 & & & \\
\hline Agostinho et al & 81.8 & 4.2 & 35 & 81.7 & 3.4 & 35 & $6.0 \%$ & $0.10[-1.69,1.89]$ & 2015 & & & \\
\hline El Aouame et al & 77.26 & 5.05 & 23 & 79.03 & 3.68 & 30 & $4.5 \%$ & $-1.77[-4.22,0.68]$ & 2016 & & E & \\
\hline Chambi-Rocha et al & 81.75 & 4.57 & 33 & 79.43 & 3.38 & 22 & $5.3 \%$ & $2.32[0.22,4.42]$ & 2018 & & & \\
\hline Chambi-Rocha 1 et al & 77.11 & 4.63 & 23 & 78.94 & 2.57 & 20 & $5.0 \%$ & $-1.83[-4.03,0.37]$ & 2018 & & 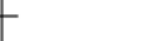 & \\
\hline Total $(95 \% \mathrm{Cl})$ & & & 606 & & & 626 & $100.0 \%$ & $-1.33[-2.03,-0.63]$ & & & & \\
\hline $\begin{array}{l}\text { Heterogeneity: } \mathrm{Tau}^{2}= \\
\text { Test for overall effect: }\end{array}$ & $\begin{array}{l}28 ; \mathrm{Ch} \\
=3.72\end{array}$ & $\begin{array}{l}=48 \\
=0\end{array}$ & , df $=$ & $=15(\mathrm{P}$ & 0.00 & $; 1^{2}=$ & $69 \%$ & & & $\begin{array}{ccc}1 & 1 & 0 \\
-4 & -2 & 0 \\
& \text { Favours [MB] }\end{array}$ & Favours [NB] & 4 \\
\hline
\end{tabular}

Figure 2. Forest plot for the variable SNA angle. SD, standard deviation; MB, mouth breathing; NB, nasal breathing; IV, inverse variance; df, degrees of freedom.

\begin{tabular}{|c|c|c|c|c|c|c|c|c|c|c|c|c|c|}
\hline \multirow{3}{*}{$\begin{array}{l}\text { Study or Subgroup } \\
\text { Bresolin et al }\end{array}$} & \multicolumn{3}{|c|}{ Mouth Breathers } & \multicolumn{3}{|c|}{ Nasal Breathers } & \multicolumn{3}{|c|}{ Mean Difference } & \multirow{2}{*}{\multicolumn{4}{|c|}{$\begin{array}{c}\text { Mean Difference } \\
\text { IV, Random, } 95 \% \mathrm{CI}\end{array}$}} \\
\hline & Mean & SD & Total & Mean & SD & Total & Weight & IV, Random, $95 \% \mathrm{Cl}$ & Year & & & & \\
\hline & 75.4 & 3.2 & 30 & 78.2 & 3.2 & 15 & $5.2 \%$ & $-2.80[-4.78,-0.82]$ & 1983 & & & & \\
\hline Faria et al & 75.8 & 3.86 & 20 & 78.43 & 2.53 & 15 & $5.0 \%$ & $-2.63[-4.75,-0.51]$ & 2002 & & & & \\
\hline Mattar et al & 76.53 & 2.8 & 44 & 77.31 & 4.99 & 29 & $5.2 \%$ & $-0.78[-2.78,1.22]$ & 2004 & & & & \\
\hline Frasson et al & 79.96 & 3.2 & 25 & 79.86 & 3.12 & 25 & $5.6 \%$ & $0.10[-1.65,1.85]$ & 2006 & & & & \\
\hline Cuccia et al & 77.1 & 3.9 & 35 & 77.55 & 3.94 & 35 & $5.5 \%$ & $-0.45[-2.29,1.39]$ & 2008 & & & & \\
\hline Juliano et al & 79.4 & 4.1 & 52 & 77.5 & 3.6 & 90 & $6.3 \%$ & $1.90[0.56,3.24]$ & 2009 & & & $\longrightarrow$ & \\
\hline Harari et al & 74.84 & 3.94 & 55 & 76.96 & 3.61 & 61 & $6.3 \%$ & $-2.12[-3.50,-0.74]$ & 2010 & & & & \\
\hline D'Ascanio et al & 75.36 & 4.89 & 98 & 79.54 & 2.18 & 98 & $6.8 \%$ & $-4.18[-5.24,-3.12]$ & 2010 & & - & & \\
\hline Malhotra et al & 74.72 & 1.36 & 20 & 75.67 & 1.87 & 18 & $6.8 \%$ & $-0.95[-2.00,0.10]$ & 2012 & & & & \\
\hline Malhotra1 et al & 76.13 & 1.9 & 34 & 79.43 & 1.74 & 28 & $7.0 \%$ & $-3.30[-4.21,-2.39]$ & 2012 & & & & \\
\hline Souki et al & 75.72 & 3.7 & 126 & 76.78 & 3.21 & 126 & $7.1 \%$ & $-1.06[-1.92,-0.20]$ & 2012 & & & & \\
\hline Ucar et al & 76.3 & 3.22 & 34 & 77.42 & 2.51 & 32 & $6.2 \%$ & $-1.12[-2.51,0.27]$ & 2012 & & & & \\
\hline Munoz et al & 76.3 & 3.9 & 53 & 77.7 & 3.6 & 65 & $6.3 \%$ & $-1.40[-2.77,-0.03]$ & 2014 & & & & \\
\hline Agostinho et al & 77.4 & 3.8 & 35 & 78 & 3.4 & 35 & $5.7 \%$ & $-0.60[-2.29,1.09]$ & 2015 & & & - & \\
\hline El Aouame et al & 73.47 & 4.86 & 23 & 73.46 & 3.57 & 30 & $4.6 \%$ & $0.01[-2.35,2.37]$ & 2016 & & & & \\
\hline Chambi-Rocha 1 et al & 77.35 & 3.81 & 33 & 77.5 & 4.5 & 22 & $4.7 \%$ & $-0.15[-2.44,2.14]$ & 2018 & & & & \\
\hline Chambi-Rocha et al & 74.25 & 3.18 & 23 & 77.68 & 2.65 & 20 & $5.6 \%$ & $-3.43[-5.17,-1.69]$ & 2018 & & & & \\
\hline Total $(95 \% \mathrm{Cl})$ & & & 740 & & & 744 & $100.0 \%$ & $-1.40[-2.20,-0.60]$ & & & & & \\
\hline \multicolumn{9}{|c|}{$\begin{array}{l}\text { Heterogeneity: } \mathrm{Tau}^{2}=2.16 ; \mathrm{Chi}^{2}=83.54, \mathrm{df}=16(\mathrm{P}<0.00001) ; \mathrm{I}^{2}=81 \% \\
\text { Test for overall effect: } \mathrm{Z}=3.43(\mathrm{P}=0.0006)\end{array}$} & & -10 & $\begin{array}{l}-5 \\
-5 \\
\text { Favours [MB] }\end{array}$ & $\begin{array}{lc}1 & 1 \\
& 5 \\
\text { Favours [NB] }\end{array}$ & 10 \\
\hline
\end{tabular}

Figure 3. Forest plot for the variable SNB angle. SD, standard deviation; MB, mouth breathing; NB, nasal breathing; IV, inverse variance; df, degrees of freedom

differences between different age groups in the samples of studies reporting positive results as compared to those reporting no difference. However, studies by Mattar et al (25), which had the youngest sample amongst all studies included (3-6 years) and El Aouame et al (29), which had the oldest sample (mean: 16 years 8 months), reported no difference in maxillary and mandibular positions between study and control groups. Similar results have been reported by other authors (35). However, the present quantitative analysis indicated a statistically significant reduction in SNA and SNB angles in mouth breathers.

The retracted position of the maxilla in mouth breathers has been explained with the functional matrix theory of Moss and Salentijn (2). It has been postulated that, in the absence of nasal respiration, there is a hypoplasia of the maxillary sinus and narrowing of nasal cavities with resultant maxillary retrognathism (24). Ricketts (36) has attributed a reduction in SNB angles in cases of nasal obstructions to a more forward and downward tongue posture to facilitate oral breathing.
This tongue posture possibly causes downward and backward positioning of the mandible. Souki et al (34) reported smaller mandibular corpus lengths in mouth breathers, which the authors suggest is responsible for smaller SNB angles in mouth breathers. Studies have also reported a greater incidence of skeletal class 2 malocclusion in mouth breathers $(33,34)$. However, the same was not corroborated in the present meta-analysis. The present analysis of the ANB angle indicates no difference between mouth breathers and nasal breathers. The variation amongst studies on SNA, SNB and ANB angles may be due to geographical, gender and age variations amongst study samples. In addition, only certain studies $(6,30)$ restricted the study sample to individuals with only skeletal class 1 malocclusion, whilst all others included a mixed population of patients, which may have influenced the results.

In terms of facial morphology, mouth breathers are expected to have a vertical growth pattern. The results of the present analysis tend to support this assumption. Comparisons of mandibular plane angles between mouth breathers and nasal 


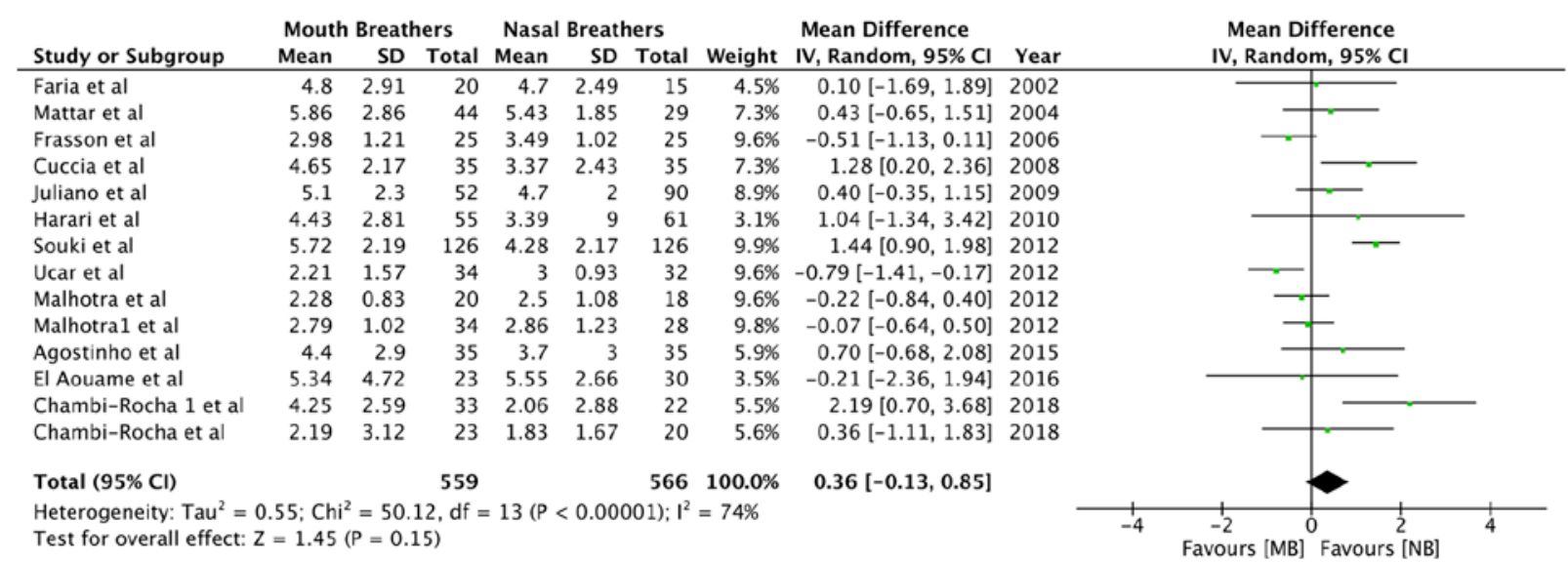

Figure 4. Forest plot for the variable ANB angle. SD, standard deviation; MB, mouth breathing; NB, nasal breathing; IV, inverse variance; df, degrees of freedom

A

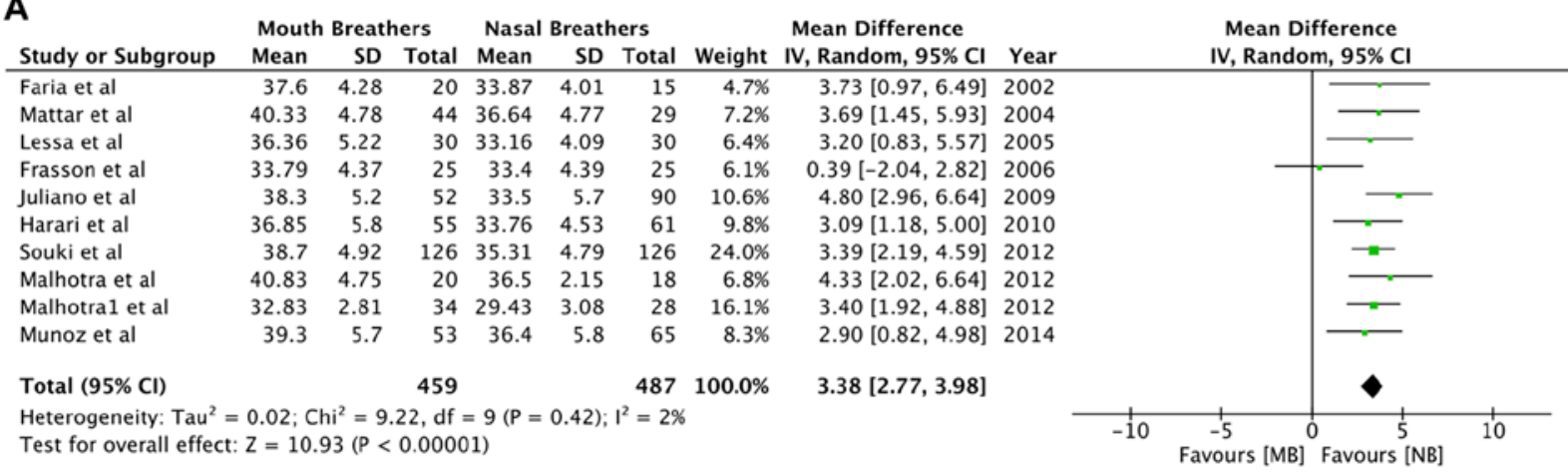

B

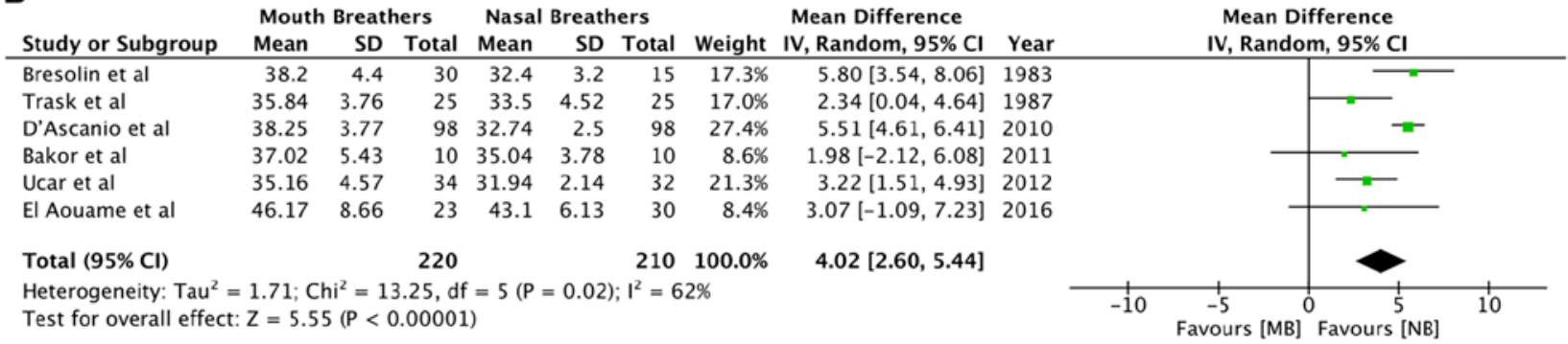

C

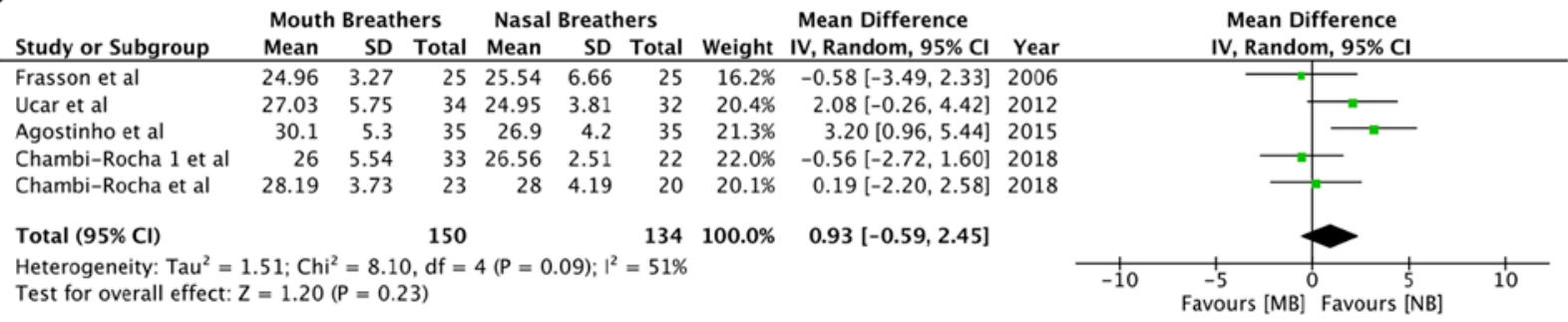

Figure 5. Forest plots for the variables (A) SN.GoGn angle, (B) FMS angle and (C) SN.MP angle. SD, standard deviation; MB, mouth breathing; NB, nasal breathing; IV, inverse variance; df, degrees of freedom.

breathers were provided by 17 studies. Since 3 different angular measurements (SN.GoGn, FMA and SN.MP) were utilized, data were pooled separately for each variable. The meta-analysis indicated a statistically significant increase in mandibular plane angles (SN.GoGn and SN.MP) in mouth breathers. Only 4 studies, namely those of Frasson et al (10), Bakor et al (3),
Chambi-Rocha et al (30) and El Aouame et al (29), did not obtain any difference in the mandibular plane angle between the two groups. In the study by Bakor et al (3), the non-significant result was probably due to the small sample size of the study. The study by El Aouame et al (29), despite no significant difference in mandibular plane angles, concluded that a 
A

\begin{tabular}{|c|c|c|c|c|c|c|c|c|c|}
\hline \multirow{2}{*}{ Study or Subgroup } & \multicolumn{3}{|c|}{ Mouth Breathers } & \multicolumn{3}{|c|}{ Nasal Breathers } & \multicolumn{3}{|c|}{ Mean Difference } \\
\hline & Mean & SD & Total & Mean & SD & Total & Weight & IV, Random, $95 \% \mathrm{CI}$ & Ye \\
\hline Faria et al & 70.4 & 4.68 & 20 & 67.8 & 2.76 & 15 & $12.1 \%$ & $2.60[0.12,5.08]$ & 2002 \\
\hline Mattar et al & 74.19 & 2.96 & 44 & 74.4 & 4.98 & 29 & $14.0 \%$ & $-0.21[-2.22,1.80]$ & 2004 \\
\hline Bakor et al & 70.18 & 3.29 & 10 & 68.72 & 2.78 & 10 & $11.5 \%$ & $1.46[-1.21,4.13]$ & 2011 \\
\hline Malhotra et al & 71.94 & 4.11 & 20 & 66.67 & 1.97 & 18 & $13.9 \%$ & $5.27[3.25,7.29]$ & 2012 \\
\hline Malhotra1 et al & 70.21 & 4.19 & 34 & 65.43 & 2.68 & 28 & $15.1 \%$ & $4.78[3.06,6.50]$ & 2012 \\
\hline Souki et al & 71.06 & 3.66 & 126 & 68.38 & 3.36 & 126 & $18.2 \%$ & $2.68[1.81,3.55]$ & 2012 \\
\hline Agostinho et al & 68.5 & 4 & 35 & 67.9 & 3.3 & 35 & $15.1 \%$ & $0.60[-1.12,2.32]$ & 2015 \\
\hline ot & & & 289 & & & 261 & $100.0 \%$ & $2.49[1.09,3.89]$ & \\
\hline
\end{tabular}

Heterogeneity: $\mathrm{Tau}^{2}=2.61 ; \mathrm{Chi}^{2}=26.30, \mathrm{df}=6(\mathrm{P}=0.0002) ; \mathrm{I}^{2}=77 \%$

Test for overall effect: $Z=3.48(P=0.0005)$

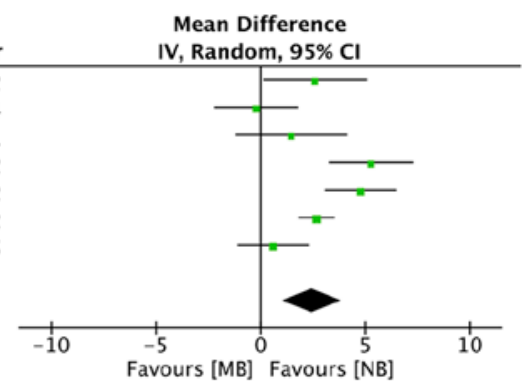

B

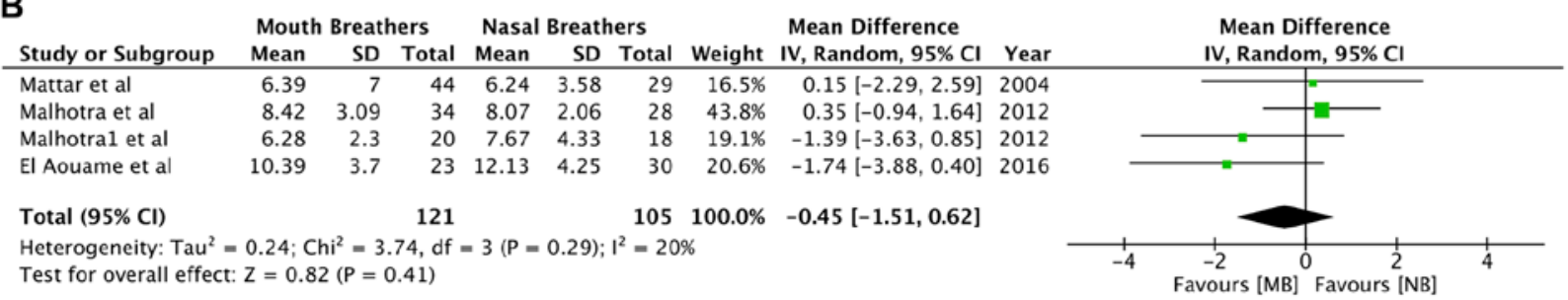

C

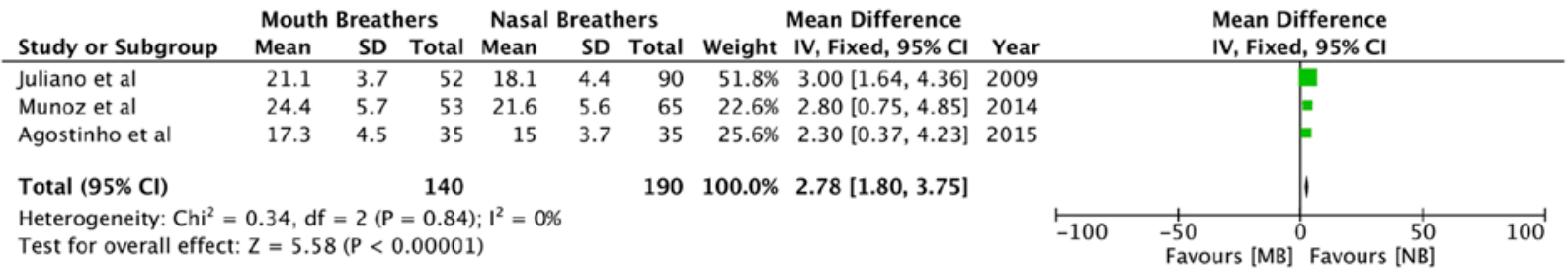

D

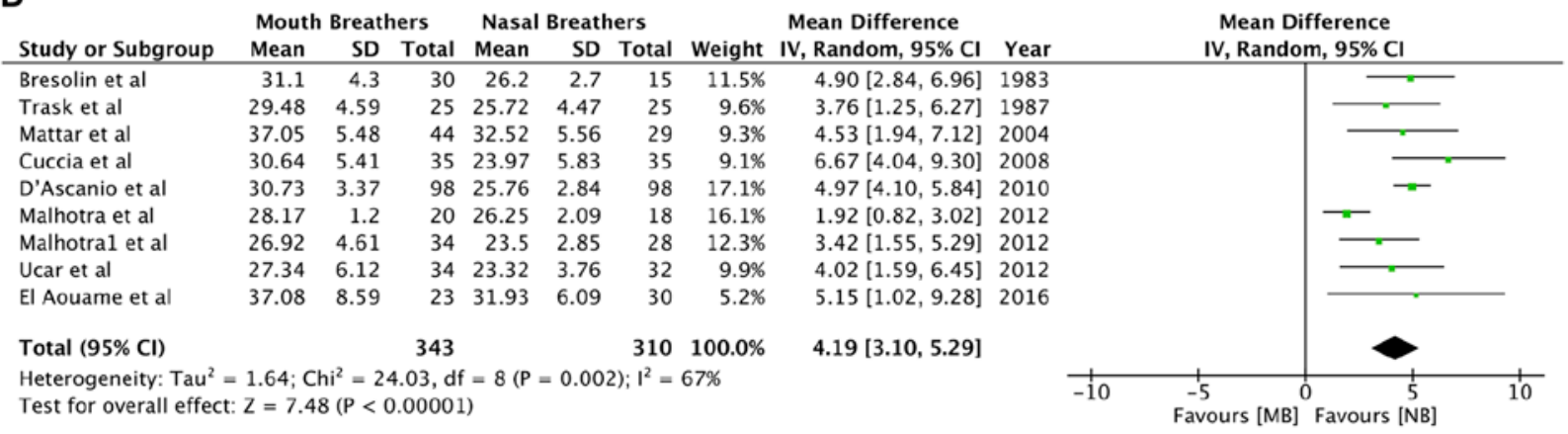

E

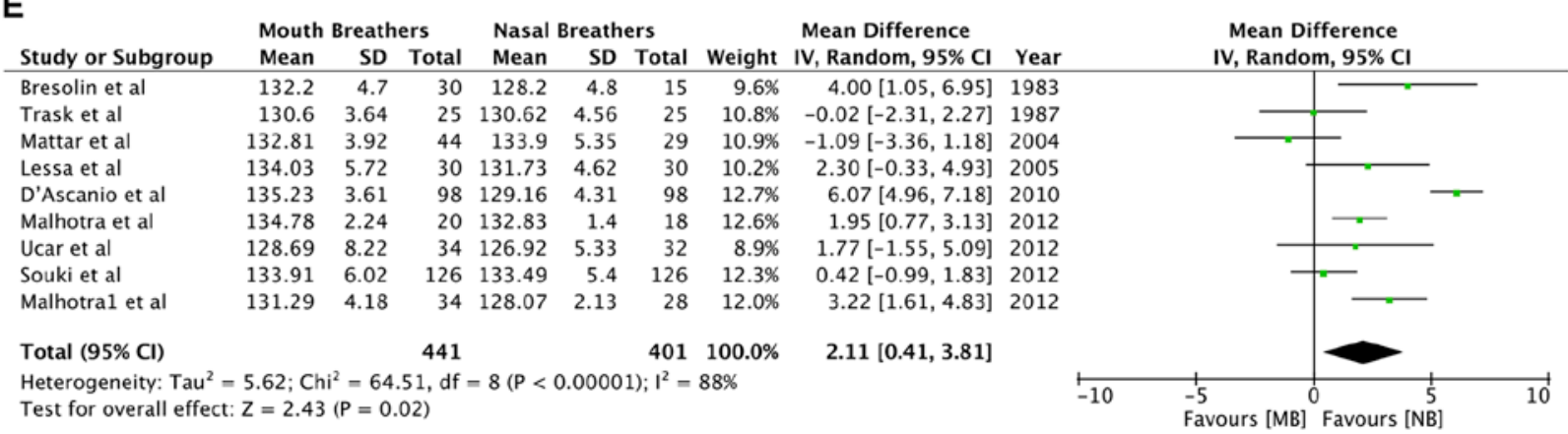

Figure 6. Forest plots for the variables (A) NSGn angle, (B) SN.PP angle, (C) SN.OP angle, (D) MP.PP angle and (E) ArGoMe angle. SD, standard deviation; $\mathrm{MB}$, mouth breathing; NB, nasal breathing; IV, inverse variance; df, degrees of freedom.

hyperdivergent growth pattern prevails in mouth breathers as compared to nasal breathers.

An increase in the Y-axis angle is also indicative of a vertical growth pattern. Analysis of data from 6 studies $(3,7,24,25,27,34)$ demonstrated increased NSGn angles in mouth breathers. The present results also revealed a significant increase in the occlusal plane angle in mouth breathers but without any difference in palatal plane angle. The mandibular plane angle was increased in association with the palatal plane in mouth breathers. Thus, the present results indicate a counterclockwise rotation of the mandible to the cranial base in mouth breathers, but without any concomitant rotation of the maxilla.

Linear variables in the present study were restricted to facial height measurements. The present results indicated a 


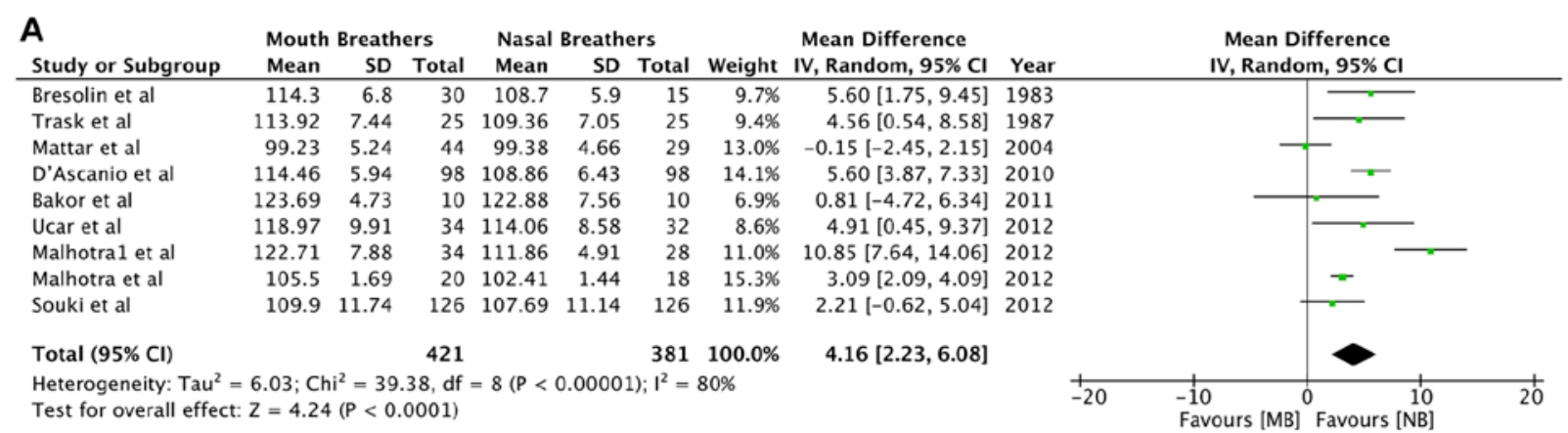

B

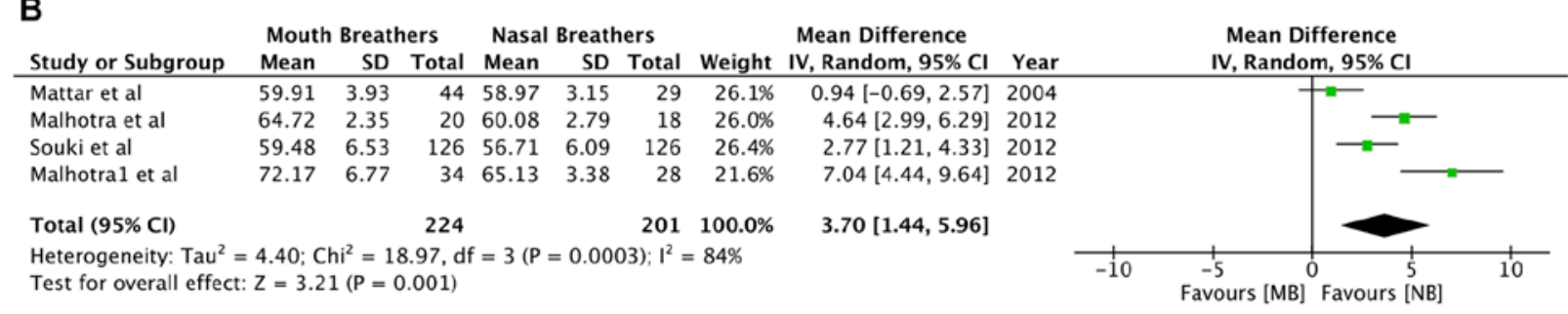

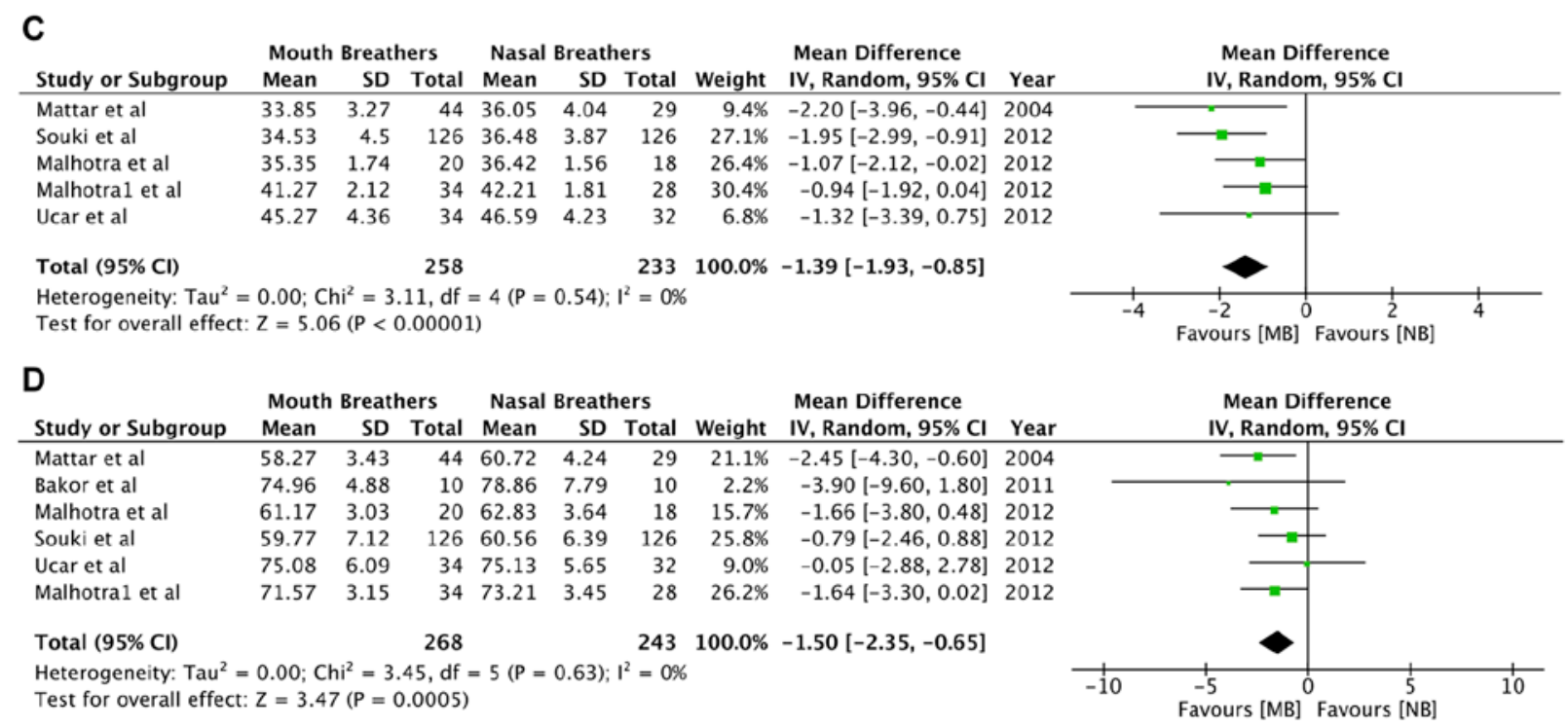

Figure 7. Forest plots for the variables (A) N-Me, (B) ANS-Me, (C) Ar-Go and (D) S-Go. SD, standard deviation; MB, mouth breathing; NB, nasal breathing; $\mathrm{IV}$, inverse variance; $\mathrm{df}$, degrees of freedom.

significant increase in total anterior facial height and lower anterior facial height in mouth breathers, accompanied by a decrease in posterior facial height. While data on total anterior facial height were reported by 8 studies $(3,6,23,25,27,31,33,34)$, values of lower anterior facial height were only available from 3 studies $(25,27,34)$. Souki et al (34) have pointed out the difference in lower anterior facial height in children with primary dentition as compared to those in the mixed dentition phase. It is contended that facial height may be influenced by the skeletal maturation stage and not by the breathing mode alone. Therefore, the ratio of posterior facial height to total anterior facial height is regarded as a better tool to understand facial morphology. In their study, no difference with regard to age groups was identified when comparing this ratio between study and control groups. The posterior-to-anterior facial height ratio was reported by a small number of studies included in the present review $(3,32,34)$. All reported values were decreased in mouth breathers as compared to nasal breathers. The variance in facial height tends to support the theory that mouth breathers exhibit clockwise rotation of the mandible with increased vertical growth of the anterior portion of the face relative to the posterior portion of the face.

Certain limitations of the present study require to be elaborated on and considered while interpreting the results. Firstly, the strength of evidence of any review and meta-analysis is measured by the quality of the included studies. Based on the present assessment, the quality of the included studies was not high. The biggest drawback is the baseline similarity assessment between study and control groups. Statistical analysis and skeletal maturity indicators were not used by the majority of studies to establish baseline similarity amongst the study groups. Not all studies utilized sufficient diagnostic tools, including nasopharyngoscopy and/or rhinomanometry, to confirm the MB habit. Furthermore, the present analysis only included data from cross-sectional studies. Long-term longitudinal studies are required to establish the exact role of $\mathrm{MB}$ in craniofacial development. In addition, the large 
variance in the age groups of individual studies combined with insufficient data precluded a sub-group analysis based on the age of participants. The studies also reported data of individuals from diverse geographical regions and ethnic backgrounds. This may have introduced bias in the results of the present review. As another limitation, only lateral cephalometric data were analyzed, as sufficient studies on posteroanterior cephalogram were not available. Similarly, the lack of sufficient studies on soft tissue data precluded a meta-analysis for soft-tissue variables. Finally, cephalometric data are prone to errors through several factors, including radiographic magnification, patient position and observer variance. Such errors across a large number of studies may have influenced the results.

However, since the present study was a study-level meta-analysis and not a patient-level study, it may be assumed that cephalometric errors were equally distributed amongst the study and control groups. Despite the drawbacks, the consistency of size and directionality of the overall effect, as well as the stability of the results after sensitivity analysis, support the reliability of the results of the present meta-analysis.

The present results indicated that mouth breathers had a tendency of retrognathic maxilla and mandible as compared to nasal breathers. The association of the maxilla with the mandible appears to be normal. Mouth breathers tend to have a high mandibular plane angle and an increased gonial angle. The tendency of downward and backward rotation of the mandible is observed in mouth breathers without a similar angular change in the maxilla. Total anterior facial height and lower anterior facial height appear to be increased while posterior facial height appears to be decreased in mouth breathers.

However, the quality of evidence is not high. More high-quality studies with longitudinal assessment of growth are required to help strengthen the evidence on this subject.

\section{Acknowledgements}

Not applicable.

\section{Funding}

This study was supported by Contract for Science and Technology Projects in Jiaxing City (community; grant no. 2018AD32035). The subject of the project is 'The Effect of Muscle Functional Appliance on Malocclusion of Tooth Collar Caused by MB in Mixed Teeth'.

\section{Availability of data and materials}

The datasets used and/or analyzed during the present study are available from the corresponding author on reasonable request.

\section{Authors' contributions}

WZ conceived and designed the study. XZ, JD and JH collected the data and performed the literature search. WZ was involved in the writing of the manuscript. All authors have read and approved the final manuscript.

\section{Ethics approval and consent to participate}

Not applicable.

\section{Patient consent for publication}

Not applicable.

\section{Competing interests}

The authors declare that they have no competing interests.

\section{References}

1. Warren DW: Effect of airway obstruction upon facial growth. Otolaryngol Clin North Am 23: 699-712, 1990.

2. Moss ML and Salentijn L: The primary role of functional matrices in facial growth. Am J Orthod 55: 566-577, 1969.

3. Bakor SF, Enlow DH, Pontes P and De Biase NG: Craniofacial growth variations in nasal-breathing, oral-breathing, and tracheotomized children. Am J Orthod Dentofacial Orthop 140: 486-492, 2011

4. Harari D, Redlich M, Miri S, Hamud T and Gross M: The effect of mouth breathing versus nasal breathing on dentofacial and craniofacial development in orthodontic patients. Laryngoscope 120: 2089-2093, 2010.

5. Sousa JB, Anselmo-Lima WT, Valera FC, Gallego AJ and Matsumoto MA: Cephalometric assessment of the mandibular growth pattern in mouth-breathing children. Int $\mathbf{J}$ Pediatr Otorhinolaryngol 69: 311-317, 2005.

6. Ucar FI, Ekizer A and Uysal T: Comparison of craniofacial morphology, head posture and hyoid bone position with different breathing patterns. Saudi Dent J 24: 135-141, 2012.

7. Agostinho HA, Furtado IÂ, Silva FS and Ustrell Torrent J: Cephalometric evaluation of children with allergic rhinitis and mouth breathing. Acta Med Port 28: 316-21, 2015.

8. Basheer B, Hegde KS, Bhat SS, Umar D and Baroudi K: Influence of mouth breathing on the dentofacial growth of children: A cephalometric study. J Int Oral Health 6: 50-55, 2014.

9. Cuccia AM, Lotti M and Caradonna D: Oral breathing and head posture. Angle Orthod 78: 77-82, 2008.

10. Frasson JM, Magnani MB, Nouer DF, de Siqueira VC and Lunardi N: Comparative cephalometric study between nasal and predominantly mouth breathers. Braz J Otorhinolaryngol 72: 72-81, 2015.

11. Fields HW, Warren DW, Black K and Phillips CL: Relationship between vertical dentofacial morphology and respiration in adolescents. Am J Orthod Dentofacial Orthop 99: 147-154, 1991.

12. Vig KW: Nasal obstruction and facial growth: The strength of evidence for clinical assumptions. Am J Orthod Dentofacial Orthop 113: 603-611, 1998.

13. Welch V, Petticrew M, Tugwell P, Moher D, O'Neill J, Waters E and White H; PRISMA-Equity Bellagio group: PRISMA-equity 2012 extension: Reporting guidelines for systematic reviews with a focus on health equity. PLoS Medicine 9: e1001333, 2012.

14. Flores-Mir C, Korayem M, Heo G, Witmans M, Major MP and Major PW: Craniofacial morphological characteristics in children with obstructive sleep apnea syndrome: A systematic review and meta-analysis. J Am Dent Assoc 144: 269-277, 2013.

15. Franco LP, Souki BQ, Pereira TB, Meyge de Brito G, Gonçalves Becker HM and Pinto JA: Is the growth pattern in mouth breathers comparable with the counterclockwise mandibular rotation of nasal breathers? Am J Orthod Dentofacial Orthop 144: 341-348, 2013.

16. Valera FC, Travitzki LV, Mattar SE, Matsumoto MA, Elias AM and Anselmo-Lima WT: Muscular, functional and orthodontic changes in pre school children with enlarged adenoids and tonsils. Int J Pediatr Otorhinolaryngol 67: 761-770, 2003.

17. Zicari AM, Albani F, Ntrekou P, Rugiano A, Duse M, Mattei A and Marzo G: Oral breathing and dental malocclusions. Eur J Paediatr Dent 10: 59-64, 2009.

18. Lione R, Buongiorno M, Franchi L and Cozza P: Evaluation of maxillary arch dimensions and palatal morphology in mouth-breathing children by using digital dental casts. Int $\mathbf{J}$ Pediatr Otorhinolaryngol 78: 91-95, 2014. 
19. Rossi RC, Rossi NJ, Rossi NJ, Yamashita HK and Pignatari SS Dentofacial characteristics of oral breathers in different ages: A retrospective case-control study. Prog Orthod 16: 23, 2015.

20. Ung N, Koenig J, Shapiro PA, Shapiro G and Trask G: A quantitative assessment of respiratory patterns and their effects on dentofacial development. Am J Orthod Dentofacial Orthop 98: 523-532, 1990

21. Jakobsone G, Urtane I and Terauds I: Soft tissue profile of children with impaired nasal breathing. Stomatologija 8: 39-43, 2006.

22. Cheng MC, Enlow DH, Papsidero M, Broadbent BH Jr, Oyen O and Sabat M: Developmental effects of impaired breathing in the face of the growing child. Angle Orthod 58: 309-320, 1988.

23. Trask GM, Shapiro GG and Shapiro PA: The effects of perennial allergic rhinitis on dental and skeletal development: A comparison of sibling pairs. Am J Orthod Dentofacial Orthop 92: 286-293, 1987.

24. Faria PT, de Oliveira Ruellas AC, Matsumoto MA, AnselmoLima WT and Pereira FC: Dentofacial morphology of mouth breathing children. Braz Den J 13: 129-132, 2002.

25. Mattar SE, Anselmo-Lima WT, Valera FC and Matsumoto MA: Skeletal and occlusal characteristics in mouth-breathing pre-school children. J Clin Pediatr Dent 28: 315-318, 2004.

26. Juliano ML, Machado MA, Carvalho LB, Prado LB and do Prado GF: Mouth breathing children have cephalometric patterns similar to those of adult patients with obstructive sleep apnea syndrome. Arq Neuropsiquiatr 67: 860-865, 2009.

27. Malhotra S, Pandey RK, Nagar A, Agarwal SP and Gupta VK: The effect of mouth breathing on dentofacial morphology of growing child. J Indian Soc Pedod Prev Dent 30: 27-31, 2012.

28. Chung Leng Muñoz I and Beltri Orta P: Comparison of cephalometric patterns in mouth breathing and nose breathing children. Int J Pediatr Otorhinolaryngol 78: 1167-1172, 2014.
29. El Aouame A, Daoui A and El Quars F: Nasal breathing and the vertical dimension: A cephalometric study. Int Orthod 14: 491-502, 2016.

30. Chambi-Rocha A, Cabrera-Domínguez ME and DomínguezReyes A: Breathing mode influence on craniofacial development and head posture. J Pediatr (Rio J) 94: 123-130, 2018.

31. Bresolin D, Shapiro PA, Shapiro GG, Chapko MK and Dassel S: Mouth breathing in allergic children: Its relationship to dentofacial development. Am J Orthod 83: 334-340, 1983.

32. Lessa FC, Enoki C, Feres MF, Valera FC, Lima WT and Matsumoto MA: Breathing mode influence in craniofacial development. Braz J Otorhinolaryngol 71: 156-160, 2005.

33. D'Ascanio L, Lancione C, Pompa G, Rebuffini E, Mansi N and Manzini M: Craniofacial growth in children with nasal septum deviation: A cephalometric comparative study. Int J Pediatr Otorhinolaryngol 74: 1180-1183, 2010.

34. Souki BQ, Lopes PB, Pereira TB, Franco LP, Becker HM and Oliveira DD: Mouth breathing children and cephalometric pattern: Does the stage of dental development matter? Int $\mathrm{J}$ Pediatr Otorhinolaryngol 76: 837-841, 2012.

35. Linder-Aronson S: Respiratory function in relation to facial morphology and the dentition. Br J Orthod 6: 59-71, 1979.

36. Ricketts RM: Forum on the tonsil and adenoid problem in orthodontics respiratory obstruction syndrome. Am J Orthod 54: 495-507, 1968.

(i) (9) This work is licensed under a Creative Commons Attribution-NonCommercial-NoDerivatives 4.0 International (CC BY-NC-ND 4.0) License. 